EPJ Web of Conferences 41, 08003 (2013)

DOI: $10.1051 /$ epjconf/20134108003

(C) Owned by the authors, published by EDP Sciences, 2013

\title{
Ultrafast proton coupled electron transfer (PCET) dynamics in 9-anthranol-aliphatic amine system
}

\author{
Hirendra N. Ghosh*1,2 ${ }^{1,}$ Katrin Adamczyk ${ }^{2}$, Sandeep Verma ${ }^{1}$, Jens Dreyer ${ }^{2}$, and Erik T. J. Nibbering ${ }^{1}$ \\ ${ }^{1}$ Radiation \& Photochemistry Division, Bhabha Atomic Research Centre, Trombay, Mumbai - 400 \\ 085, INDIA, Email: hnghosh@barc.gov.in \\ ${ }^{2}$ Max Born Institute for Nonlinear Optics and Short Pulse Spectroscopy, Max Born Strasse 2A, D- \\ 12489, Berlin, Germany, Email: nibberin@mbi-berlin.de
}

\begin{abstract}
Femtosecond infrared absorption studies strongly suggest that photoexcited 9-anthranol takes part in an ultrafast electron transfer (ET) reaction in electron-donating triethylamine solvent, but that ultrafast proton coupled electron transfer (PCET) occurs in diethylamine solvent.
\end{abstract}

\section{Introduction}

Proton-coupled electron transfer (PCET) is one of the most fundamental reactions and play important roles in energy conversion in biological systems, including water oxidation at the oxygen evolving center in photosynthesis [1] and four-electron reduction of dioxygen by cytochrome c oxidase in respiration [2]. A large number of experimental and theoretical investigations have been carried out to unravel the mechanism of PCET in many chemical and biological systems [3, 4]. Time-resolved spectroscopic techniques have been employed by many authors which have demonstrated PCET reactions taking place on micro- and milisecond time scales, where in most of the cases the mechanism is not clearly understood. To understand the underlying mechanisms and energetics of PCET reactions it is highly demandable to investigate such processes using model systems on ultrafast time scales, where so far, however, not many examples have been reported in the literature. Here we report on the electron transfer (ET) reaction between photoexcited 9-anthranol (ANTH) and the aliphatic amines diethylamine (DEA) and triethylamine (TEA) using femtosecond IR absorption spectroscopy. In addition to the ET reaction we also observe a proton transfer, thus making it a PCET reaction, for the ANTH-DEA reaction pair. Our observations are supported by steady-state and transient fluorescence emission and femtosecond visible absorption studies and DFT calculations.

\section{Results and Discussion}

Figure 1A shows optical absorption spectra of 9-anthrone/9-anthranol in tetrachloroethylene (TCE, non-interacting solvent), DEA and TEA (electron donating solvents). Freiermuth et al. [5] reported that 9-anthrone/9-anthranol exist in different tautomeric forms, namely like 9-anthrone (ANT) in TCE and acetonitrile, but 9-anthranol (ANTH) in aliphatic amine solvents, such as DEA and TEA, whereas both tautomers are present in alcoholic solvents. The presence of ANTH form in amine solvents is clearly confirmed by the appearance of a new band in $330-450 \mathrm{~nm}$ region of the optical

This is an Open Access article distributed under the terms of the Creative Commons Attribution License 2.0, which permits unrestricted use, distribution, and reproduction in any medium, provided the original work is properly cited. 
spectrum. Figure 1B shows IR absorption bands in the $1580-1620 \mathrm{~cm}^{-1}$ region which can be attributed to the $\mathrm{C}=\mathrm{C}$ ring stretching vibrational modes. The band at $1660-1690 \mathrm{~cm}^{-1}$ can be attributed to the $\mathrm{C}=\mathrm{O}$ stretching vibration for $\mathrm{ANT} / \mathrm{ANTH}$. Steady-state emission quenching and fluorescence time-resolved experiments confirmed that a photoinduced ET reaction occurs for the ANTH/amine systems, where ANTH act as electron acceptor and both the amines (DEA \& TEA) act as electron donor.
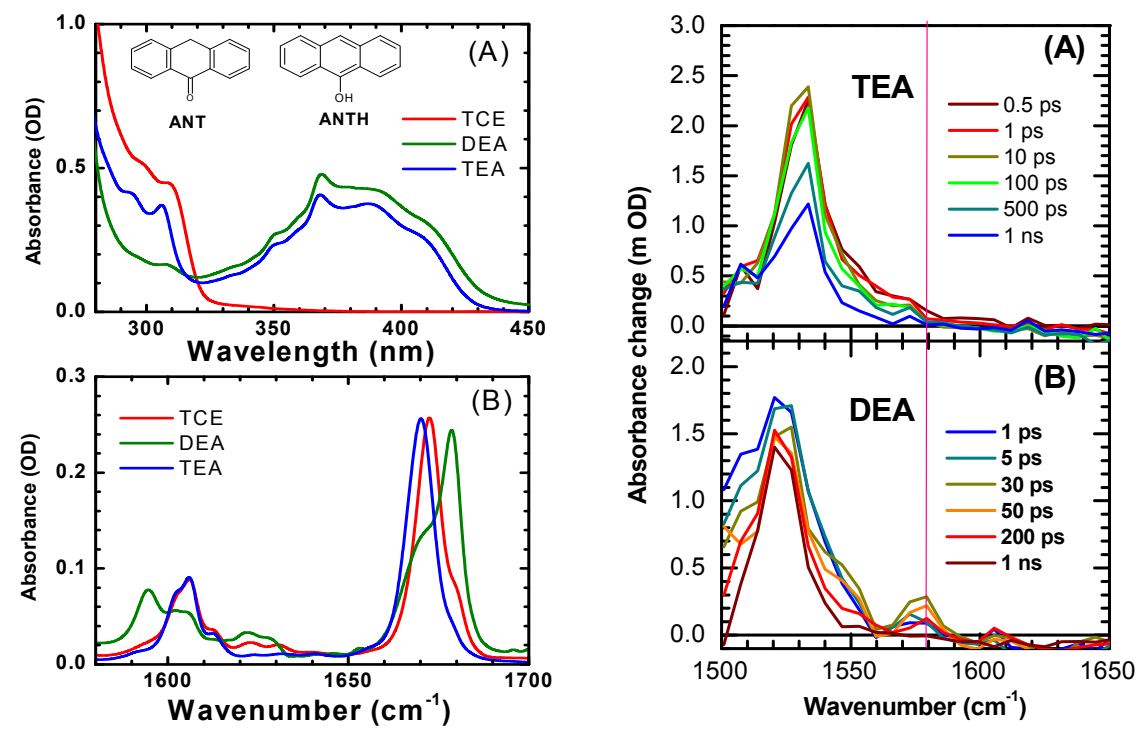

Fig. 1. UV-vis absorption spectra (A) and Fig 2. Transient IR absorption spectra of ANTH IR spectra (B) of the acceptor molecule in (A) TEA and in B) DEA at different time ANT/ANTH in different solvents: TCE delays after excitation at $400 \mathrm{~nm}$. (non interacting solvent), and electron donating TEA and DEA

To unravel the dynamics in early time domain ultrafast transient absorption studies have been carried out by pumping ANTH at $400 \mathrm{~nm}$ laser light and probing vibrational marker modes in the mid-IR region (Figure 2). Upon photoexcitation of ANTH/TEA and ANTH/DEA systems, we observe a prominent transient absorption band at $1532 \mathrm{~cm}^{-1}$ which can be attributed to the anion radical of ANTH $\left(\mathrm{ANTH}^{\bullet-}\right.$ ) (Figure 2). Interestingly, with increasing time delay a new transient absorption band appears at $1580 \mathrm{~cm}-1$ and also a hump at $1553 \mathrm{~cm}^{-1}$ in addition to the existing 1527 $\mathrm{cm}^{-1}$ band (due to $\mathrm{ANTH}^{\bullet-}$ ) in DEA(Figure 2B). This evolution of spectral features in the transient mid-IR spectra is absent in the ANTH/TEA system. Figure 3 shows the kinetics decay traces of the transients at different frequencies for both ANTH/TEA and ANTH/DEA systems. Transient kinetics at $1527 \mathrm{~cm}^{-1}$ and $1553 \mathrm{~cm}^{-1}$ for ANTH/TEA system are fitted with a $>1$ ns time constant (major component), attributed to a back ET reaction in TEA (Figure 3A). Interestingly in the ANTH/DEA system the kinetics at $1527 \mathrm{~cm}^{-1}$ can be fitted with a $50 \mathrm{ps}$ and a $>1 \mathrm{~ns}$ components. However, at $1553 \mathrm{~cm}^{-1}$ and $1580 \mathrm{~cm}^{-1}$ the growth kinetics can be fitted with a $\sim 50 \mathrm{ps}$ and the decay kinetics can be fitted with a $\sim 200$ ps component (Figure 3B). Interestingly the decay time (50 ps) at $1527 \mathrm{~cm}^{-1}$ and growth time at $1553 \mathrm{~cm}^{-1}$ and $1580 \mathrm{~cm}^{-1}$ exactly matches, which can be attributed to a proton transfer event between $\mathrm{ANTH}^{\bullet-}$ and the cation radical of DEA $\left(\mathrm{DEA}^{\bullet+}\right)$ leading to the formation of a ketyl radical of ANTH (Scheme 1). The decay constant ( 200 ps) at $1580 \mathrm{~cm}^{-1}$ can be attributed to the recombination dynamics of ANTH ketyl radical and the DEA di-radical. Additional support for this PCET reaction mechanism comes from the observation of an H/D isotope effect observed in the ANTH/DEA system. 

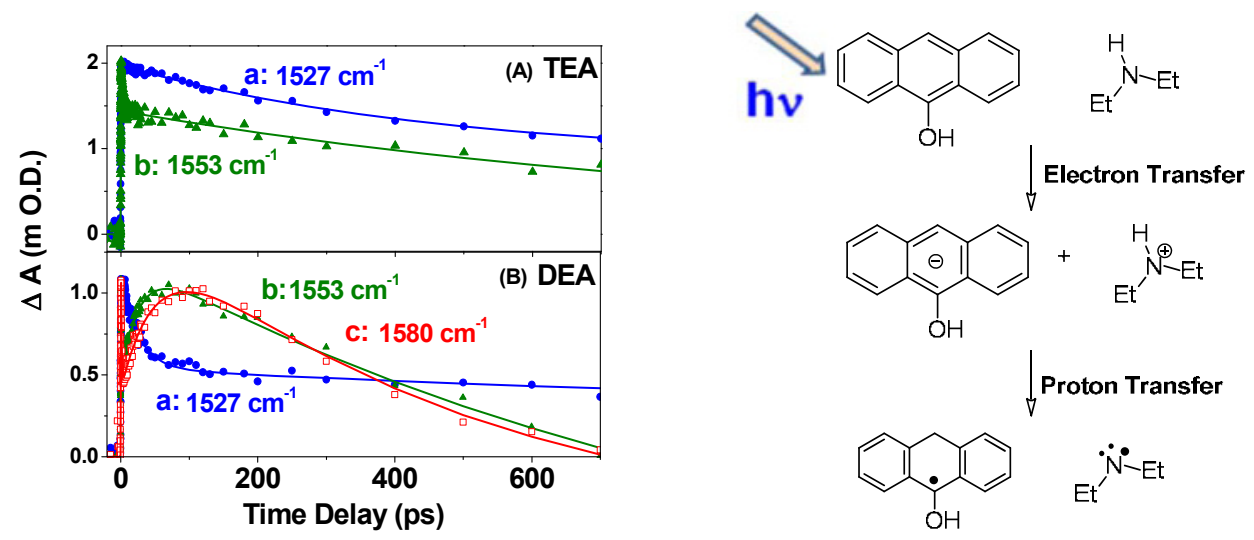

Fig. 3. Normalized transient absorption Scheme 1: Schematic diagram of PCET kinetics in (A) TEA and in (B) DEA at reaction in ANTH/DEA system. different frequencies: a) $1527 \mathrm{~cm}^{-1}$, b) 1553 $\mathrm{cm}^{-1}$, c) $1580 \mathrm{~cm}^{-1}$.

\section{Conclusions}

Ultrafast transient IR and visible absorption spectroscopy and steady state and time-resolved emission measurements have been carried out to investigate electron transfer dynamics between ANT/ANTH and aliphatic amine systems. Our experimental observations confirmed that an ultrafast electron transfer reaction takes place between the photoexcited ANTH acceptor and the amine donors (both TEA and DEA). Interestingly proton transfer has been observed only between ANTH anion and DEA cation, leading to the formation of a ketyl radical, as suggested by the observation of an H/D isotope effect. PT reaction was absent in ANTH/TEA system. To the best of our knowledge this is the first report of a proton coupled electron transfer (PCET) reaction between PCET donoracceptor pairs on ultrafast time scales.

\section{References}

1. C. W. Hoganson, G. T. Babcock, Science 277, 1953 (1997).

2. G. T. Babcock, Proc. Natl. Acad. Sci. U.S.A. 96, 12971. (1999).

3. J. M. Mayer, Annu. Rev. Phys. Chem. 55, 363 (2004).

4. M. H. V. Huynh, T.J. Meyer, Chem. Rev. 107, 5004 (2007).

5. B. Freiermuth, B. Hellrung, S. Peterli, M.-F. Schultz, D.Wintgens, J.Wirz, Helv. Chim. Act. 84, 3796 (2001). 